\title{
Beyond the Purcell Effect: Modifying Spontaneous Emission via Plasmonic Doping
}

Boyang Ding ( $\nabla$ boyang.ding@coherent.com.au )

University of Otago

Yu-Hui Chen

Beijing Institute of Technology

Mohsin ljaz

University of Otago

Isabella Wagner

Victoria University of Wellington https://orcid.org/0000-0001-5009-2407

Fengjiang Liu

Westlake University

Margarita Samoli

Ghent University https://orcid.org/0000-0003-0442-5420

\section{Zeger Hens}

Ghent University https://orcid.org/0000-0002-7041-3375

Pieter Geiregat

Ghent University https://orcid.org/0000-0001-7217-8738

Justin Hodgkiss

Victoria University of Wellington

Richard Blaikie

University of Otago

Kai Chen

Victoria University of Wellington

Min Qiu

Westlake University https://orcid.org/0000-0002-4613-5125

Physical Sciences - Article

Keywords:

Posted Date: February 14th, 2022

DOI: https://doi.org/10.21203/rs.3.rs-1243208/v1 
License: (c) (i) This work is licensed under a Creative Commons Attribution 4.0 International License. Read Full License 


\title{
Beyond the Purcell Effect: Modifying Spontaneous Emission via Plasmonic Doping
}

\author{
Yu-Hui Chen, ${ }^{1, *}$ Mohsin Ijaz, ${ }^{2,3,4, *}$ Isabella Wagner, ${ }^{2,3,5}$ Fengjiang Liu, ${ }^{6,7}$ \\ Margarita Samoli, ${ }^{8,9}$ Zeger Hens, ${ }^{8,9}$ Pieter Geiregat, ${ }^{8,9}$ Justin M. Hodgkiss, ${ }^{2,3,5}$ \\ Richard J. Blaikie,,$^{2,3,4}$ Boyang Ding,,$^{2,3,4, \dagger}$ Kai Chen,,$^{2,3,5, \ddagger}$ and Min Qiu ${ }^{6,7, \S}$ \\ ${ }^{1}$ Key Laboratory of advanced optoelectronic quantum architecture and measurements of Ministry of Education, \\ Beijing Key Laboratory of Nanophotonics \& Ultrafine Optoelectronic Systems, \\ School of Physics, Beijing Institute of Technology, Beijing 10081, China \\ ${ }^{2}$ Dodd-Walls Centre for Photonic and Quantum Technologies, New Zealand \\ ${ }^{3}$ MacDiarmid Institute for Advanced Materials and Nanotechnology, New Zealand \\ ${ }^{4}$ Department of Physics, University of Otago, PO Box 56, Dunedin 9016, New Zealand \\ ${ }^{5}$ School of Chemical and Physical Sciences, Victoria University of Wellington, Wellington 6012, New Zealand \\ ${ }^{6}$ Key Laboratory of $3 D$ Micro/Nano Fabrication and Characterization of Zhejiang Province, \\ School of Engineering, Westlake University, 18 Shilongshan Road, Hangzhou 310024, Zhejiang Province, China \\ ${ }^{7}$ Institute of Advanced Technology, Westlake Institute for Advanced Study, \\ 18 Shilongshan Road, Hangzhou 310024, Zhejiang Province, China \\ ${ }^{8}$ Physics and Chemistry of Nanostructures, Ghent University, 9000 Ghent, Belgium \\ ${ }^{9}$ Center for Nano and Biophotonics (NB Photonics), Ghent University, 9000 Ghent, Belgium
}

(Dated: January 9, 2022) 
Optical resonators can alter quantum emitters' electromagnetic environment, thus modifying the spontaneous emission[1]. This is known as the Purcell effect[2], widely regarded as the standard explanation of cavity-emitter interations. Here we show that this effect fails to properly address the emission modified by plasmonic resonators, i.e. a special type of metal cavities, where resonators can strongly affect the transition processes by charging the emitters[3]. In particular, by integrating quantum dots (QDs) into a grating-like plasmonic resonator, we can transiently dope the QDs with a large amount of hot electrons that are produced during plasmon excitation. The doping makes the excited carriers greatly outnumber the absorbed photons, forming unusal "asymmetric excitation" in individual QDs and yielding high-frequency radiative emission that can not be enabled by the Purcell enhancement. Our finding identifies a new cavity-emitter interaction pathway, initiating riveting opportunities for both fundamental studies and practical applications in laser[4-6], photovoltaics[7], and photocatalysis[8].
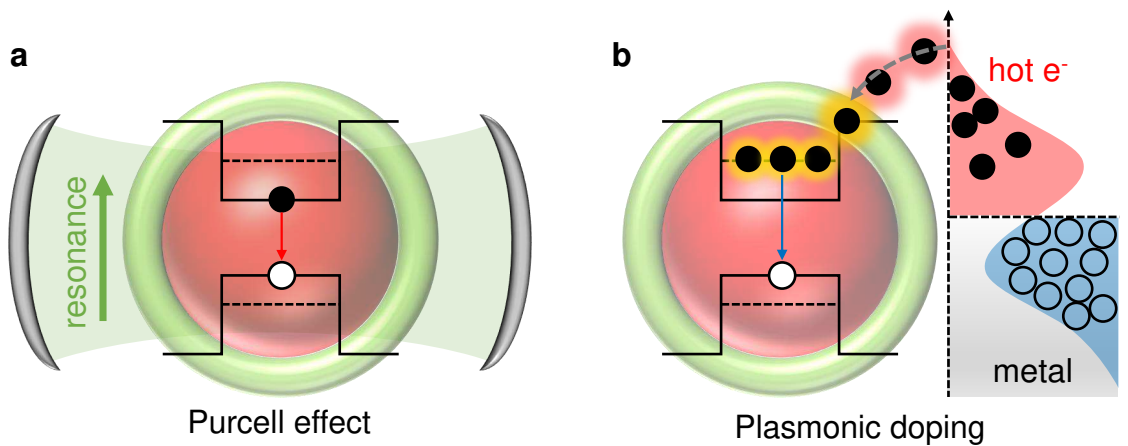

FIG. 1. Modifying emission via plasmonic doping. a, schemetic of a quantum emitter that is integrated with a conventional optical resonator, in which the interaction with photons is enhanced via the Purcell effect, presenting as one excited electron associated with one absorbed photon; note the number of excited electrons in each QD equals the number of absorbed photons, i.e. $N_{\mathrm{e}}=N_{\mathrm{ph}}$. b, schematic demonstrating the coupling of an emitter with a plasmonic resonator, in which the excitation of one plasmon can dephase to a lot more hot electrons that can dope the emitter, forming an asymmetric excitation $N_{\mathrm{e}}>N_{\mathrm{ph}}$.

Quantum emitters, like atoms, QDs and moleclues, typically have sizes much smaller than their working wavelengths, making them inherently difficult to interact with photons. As proposed by Purcell[2], one can use optical resonators to enhance the emitter-photon interaction, because resonators can confine photons, both spatially which increases the photon density near emitters, and temporally which gives the photons longer to cause an effect. As a result, one can increase the local density of optical states (LDOS), enhancing the emitters' excitation/emission rate (Fig. 1a). However, the theory of the Purcell effect was developed on electrically insulated resonators, such 
as Fabry-Perot cavities[9], whispery-gallery-mode cavities[10] and photonic-crystal cavities[11], where the resonant energy is purely photonic, restricting the effect's scope merely to the interaction between emitters and their electromagnetic environment.

In this letter, we demonstrate that when emitters are integrated with a plasmonic resonator, the resonator can modify spontaneous emission by transferring charges to the emitters. In particular, plasmonic resonators are specially designed metallic nanostructures, in which optical resonances arise due to collective oscillation of conduction-band electrons, known as plasmons. The excitation of plasmons highly concentrates optical energy, leading to significant enhancement of LDOS[1214], which makes plasmonic resonators an important platform for emission modification[15-19]. On the other hand, the dephasing of plasmons produces a number of highly energetic carriers[20] (See Fig. 1b and Supplementary Fig. S5). These so-called plasmonic hot electrons[21, 22], when doped in adjacent emitters[23], can make the number of excited carriers in individual emitters, i.e. the excitation density $\left(N_{\mathrm{e}}\right)$, exceed the absorbed photon density $\left(N_{\mathrm{ph}}\right)$, forming asymmetric excitations in emitters (Fig. 1b) and leading to emission modification that cannot be enabled by the Purcell effect.

We studied this effect on cadmium-selenide/cadmium-sulfide (CdSe/CdS) core/shell QDs[24] films that are coated on a silver (Ag) made periodic nano-line array plasmonic resonator (Fig. 2a). The line-array has a period of $\sim 500 \mathrm{~nm}$, enabling lattice plasmon modes that can be dispersively excited across the visible spectral range. As shown in Fig. 2c, under p-polarized [orientation of electric fields parallel to the plane (x-z) of incidence] illuminaton at $\theta=37^{\circ}$, the steady-state reflection spectrum of the QD-resonator hybrid displays one minimum at $2.57 \mathrm{eV}$, indicating that photons at this frequency are absorbed mainly due to plasmon excitaton. When illuminated at $\theta=$ $23^{\circ}$, the plasmon resonances red-shift, presenting one minimum at $2.41 \mathrm{eV}$. (See Supplementary Fig. S3 for the plasmon dispersion)

The plasmonic resonator enhances LDOS, which can be characterised by the Purcell factor $\left(F_{\mathrm{P}}=\gamma_{\mathrm{res}} / \gamma_{0}\right)$, where $\gamma_{\mathrm{res}}\left(\gamma_{0}\right)$ is the excitation/emission rate of emitters embedded in resonators (free-space). Fig. $2 \mathbf{b}$ shows the modelled spatial map of $F_{\mathrm{P}}$ at $E=2.41 \mathrm{eV}$, while $F_{\mathrm{P}}$ at other plasmon frequncies exhibits similar distributions and magnitudes, indicating that the dispersive plasmon modes are spectrally separated but spatially overlapped. This property makes the plasmonic resonator capable of enhancing both the QDs' absorption to the pump (by a factor of $F_{\mathrm{P}}^{\mathrm{abs}}$ ) and quantum yield to the emission (by a factor of $F_{\mathrm{P}}^{\mathrm{em}}$ ) in photoluminescence (PL) measurements. Specifically, after being pumped $(2.33 \mathrm{eV})$, the bare QD sample (Fig. 2d), i.e. the QD film de- 

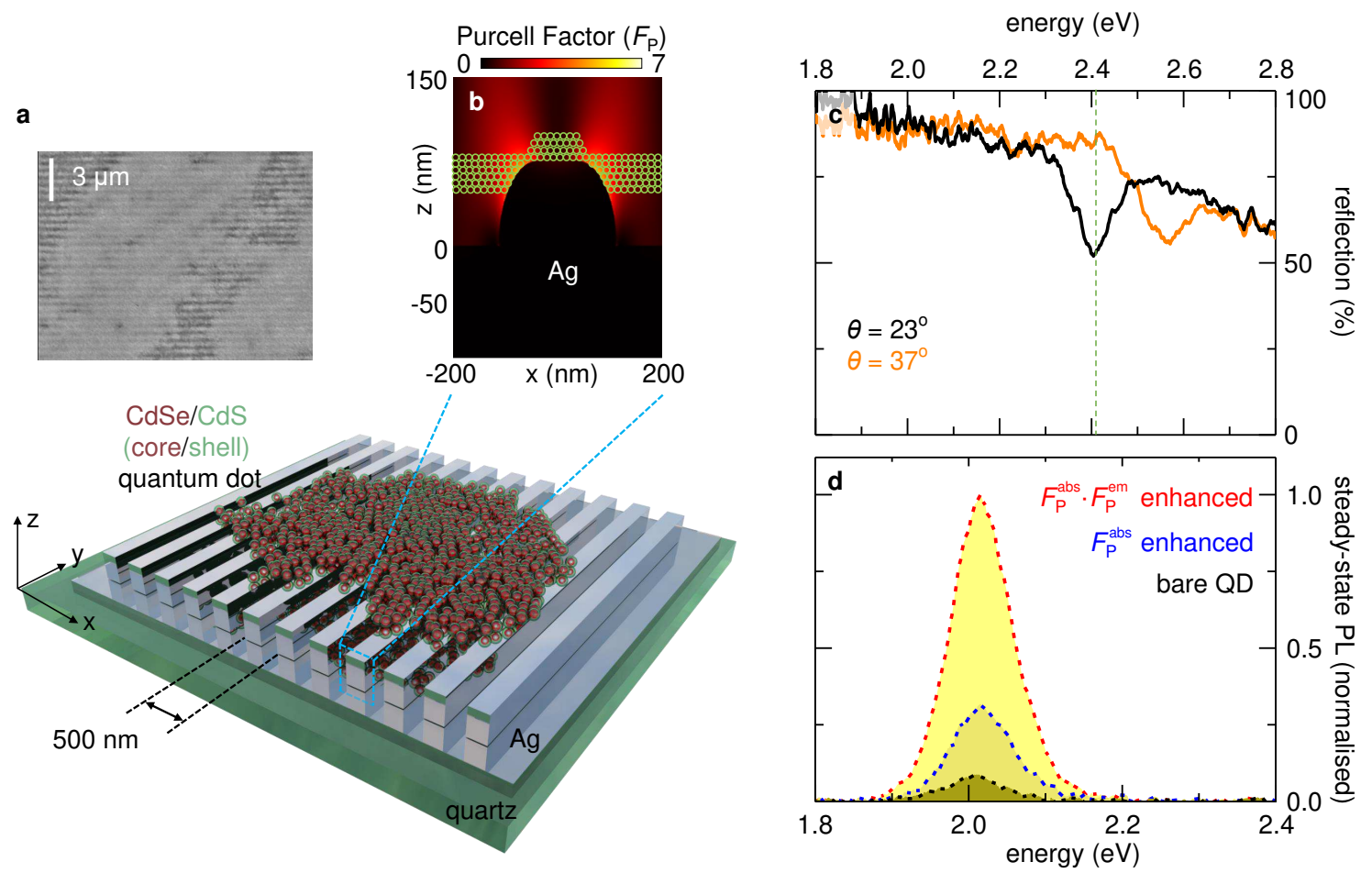

FIG. 2. Structure and steady-state optical properties. a, schematic of the QD-resonator hybrid with the scanning-electron-microscopy image and $\mathbf{b}$, the modelled spatial map of the Purcell factor along the $\mathrm{x}-\mathrm{Z}$ cross-section. More structral information can be found in Supplementary Fig. S2. c, steady-state reflection spectra under p-polarized illumination at the incident angles of $\theta=23^{\circ}$ (black) and $37^{\circ}$ (orange), respectively. The green dashed vertical line indicates the pump freuqency in the transient PL measurement (Fig. 3), which matches the plasmon resonance excited at $23^{\circ}$. d, steady-state PL spectra of bare QDs (black), only absorption $\left(F_{\mathrm{P}}^{\mathrm{abs}}\right)$ enhanced QDs (blue) and both absorption and emission $\left(F_{\mathrm{P}}^{\mathrm{abs}} \cdot F_{\mathrm{P}}^{\mathrm{em}}\right)$ enhanced QDs (red).

posited on a quartz substrate, shows a steady-state PL spectrum peaking at $2.03 \mathrm{eV}$. As shown in Fig. 2(d), when only is the absorption (at the pump frequency) enhanced, the PL in the QDresonator hybrid exhibits a 5-fold PL increase compared to the bare QDs; when both absorption and emission are enhanced, the PL can be elevated up to 20 -fold. These results agree well with the simulated $F_{\mathrm{P}}$. Please see Supplementary Fig.S8 for more information about the PL enhancement induced by $F_{\mathrm{P}}^{\mathrm{abs}}$ and $F_{\mathrm{P}}^{\mathrm{em}}$.

To further explore how the plasmon excitation affects the spontaneous emission, we studied the transient PL spectra in the QD-resonator hybrid. In our tansient gate PL spectrometer (Supplementary Fig. S1), the pump pulse has a frequency of $2.41 \mathrm{eV}(515 \mathrm{~nm})$ and a duration of $\sim 100 \mathrm{fs}$, allowing us to highly resolve the relaxation dynamics of the excited carriers, given that our QDs have a long lifetime ( $15 \mathrm{~ns})[25]$. As shown in Fig. 2c, pumping the hybrid at different angles enables selective excitation of the plasmon that is on- or off-resonance with the pump. Specifi- 

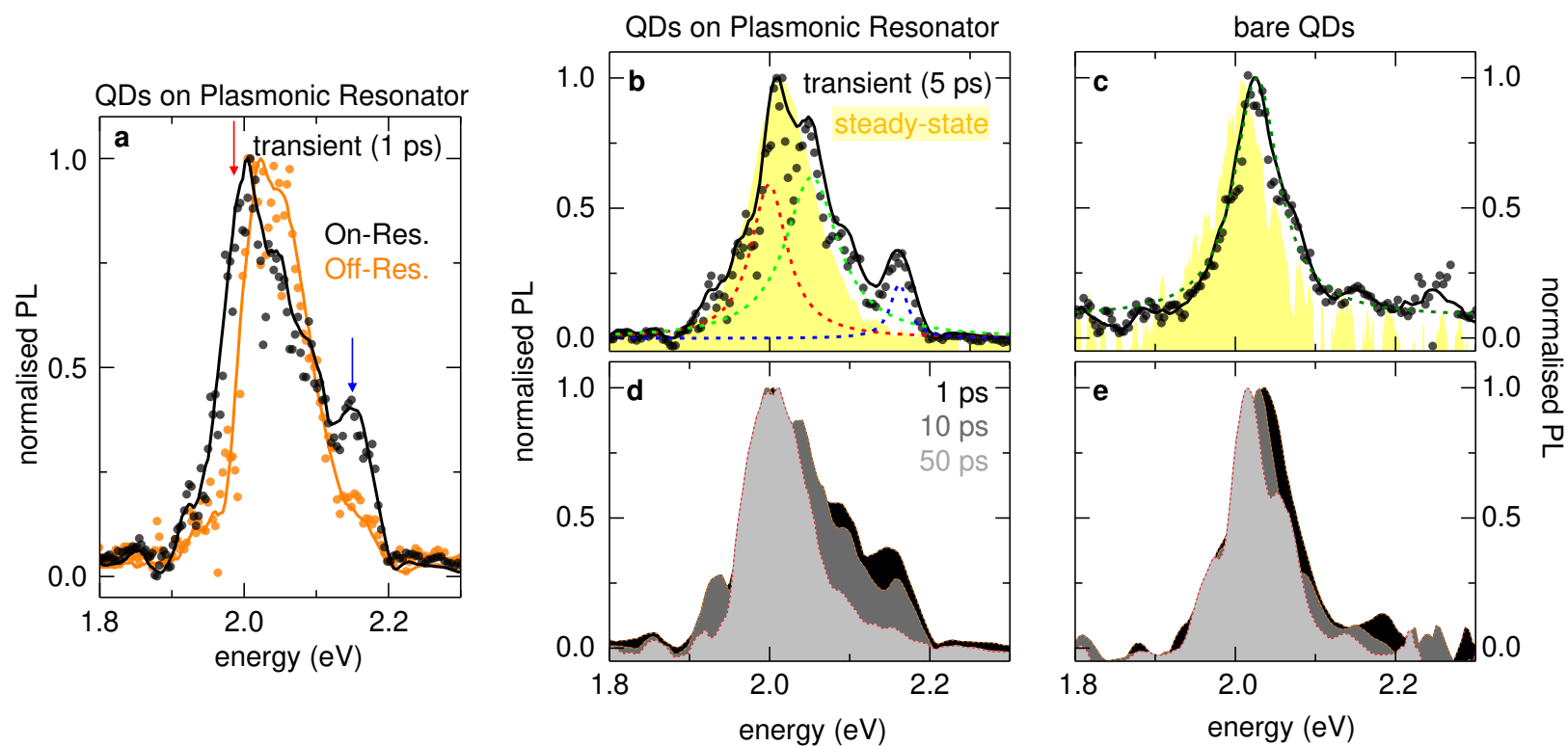

FIG. 3. Transient photoluminescence spectra. a, normalised PL transient at $t=1 \mathrm{ps}$ measured from the QD-resonator hybrid excited by the pump that is (On-Res) or not (Off-Res) plasmonically enhanced; normalised PL transient at $t=5 \mathrm{ps}$ measured from $\mathbf{b}$, the QD-resonator hybrid and $\mathbf{c}$, the bare QDs comparing with their steady-state PL (yellow area); solid circles in panel $\mathbf{a}, \mathbf{b}$ and $\mathbf{c}$ represent the raw data of the measured transient PL, where solid curves are the smoothed results; thin dashed curves in panel $\mathbf{b}$ and $\mathbf{c}$ represent the fitting components using Gaussian functions; normalised PL transients measured from d, the QD-resonator hybrid and e, the bare QDs at different times, presented with areas from black to grey.

cally, with a pulse fluence of $\sim 25.6 \mu \mathrm{J} \mathrm{cm}^{-2}$ (Fig. 3a), the on-resonance plasmon excitation (black curve) makes the PL transient ( $t=1 \mathrm{ps}$ after pumping) broadened compared to that excited by off-resonance pumping, exhibiting two pronounced additional maxima at $\sim 2.0$ and $\sim 2.15 \mathrm{eV}$ respectively (See Supplementary Fig. S7 for all PL transients). That is to say, the transient emisson spectra are modified when the pump is plasmonically enhanced.

Comparing the transient ( $t=5 \mathrm{ps}$ ) with the steady-state PL (Fig. 3b), we note the highfrequeuncy maximum at $E=2.15 \mathrm{eV}$ still presents, decaying within $50 \mathrm{ps}$ (Fig. 3d). In contast, the PL spectra (Fig. 3c and 3e) in bare QDs, irrespective of being transient or steady-state, show no sign of any high-frequency maxima. Such a high-frequency PL maximum is a clear evidence of large-density population of carriers that leads to the occupation of higher frequency states[26, 27] in QDs, thus yielding blue-shifted emission with repsect to the the $\sim 2.0 \mathrm{eV}$ bandedge transition. However, such a population in bare QD samples typically requires intense pumps that have fluences $2-3$ orders of magnitude higher than that in our experiments [25]. Given that the highfrequency maximum only appears when the pump is plasmonically enhanced, we conclude that 
the plasmon excitation at the pump frequency is responsible for the high-density population. To confirm our observations, we have also measured the PL with different fluences (Supplementary Fig. S9), in which the high-frequency maximum always exists and gets broadened with pump fluences increased from 25.62 to $256.12 \mu \mathrm{J} \mathrm{cm}^{-2}$ in our experiment.

Absorption enhancement via the Purcell effect (Fig. 1a) can certainly increase the carrier density, which, however, is far from enough to proivde the required population in high frequency states of the QDs. As shown in Fig. 2b, the pump absorption in the QDs can be enhanced up to 6-fold. However, even if pumping the bare QD sample with a 10-fold stronger fluence $\left(256.2 \mu \mathrm{J} \mathrm{cm}^{-2}\right)$, we are still unable to see any pronounced high-frequency features (Supplementary Fig. S9). According to our previous work[25], pumps $(515 \mathrm{~nm})$ that have similar fluences can only generate $<1$ excitations in individual QDs. This means that merely can the $1 \mathrm{~S}_{\mathrm{e}}$-level but not higher QD states (Supplementary Fig. S10) be filled under such relatively low fluences, and there must be other excitation mechanisms than the Purcell effect that enable the high-density population.

To figure out the excitation mechanism that enables this high-frequency emission, we need to identify the specific energy levels between which the transition can take place. The $1 \mathrm{P}_{\mathrm{e}} \rightarrow$ $1 \mathrm{P}_{3 / 2}$ transition (Supplementary Fig. S10) can be first excluded due to the frequency mismatch, which should occur at $E=2.48 \mathrm{eV}[25]$, even higher than the pump frequency. The $1 \mathrm{~S}_{\mathrm{e}} \rightarrow 1 \mathrm{P}_{3 / 2}$ and $1 \mathrm{~S}_{\mathrm{e}} \rightarrow 2 \mathrm{~S}_{3 / 2}$ transitions are close to $\sim 2.15 \mathrm{eV}$ [28], which, however, all require ultrahigh excitations to happen. In particular, to trigger the emission, one needs $\geqslant 5(\geqslant 9)$ carriers excited in individual QDs to enable the recombination of electrons and holes for the $1 \mathrm{~S}_{\mathrm{e}} \rightarrow 1 \mathrm{P}_{3 / 2}\left(1 \mathrm{~S}_{\mathrm{e}} \rightarrow\right.$ $\left.2 \mathrm{~S}_{3 / 2}\right)$ transition, which, in other words, means $\geqslant 5(\geqslant 9)$ photons must be absorbed by a single QD. Our calculation (Supplementary Eq. S1) shows that even with the maximum Purcell enhancement of the pump considered, the absorbed photon density is only $N_{\mathrm{ph}}<1$. Therefore we can rule out the possibility that the $2.15 \mathrm{eV}$ PL maximum arises due to the $1 \mathrm{~S}_{\mathrm{e}} \rightarrow 1 \mathrm{P}_{3 / 2}$ and $1 \mathrm{~S}_{\mathrm{e}} \rightarrow 2 \mathrm{~S}_{3 / 2}$ transitions. In addition, the transition between $1 \mathrm{~S}_{\mathrm{e}}$ and the thermally occupied hole states can also be excluded, because the thermal fluctuations in our system are not strong enough $\left(k_{\mathrm{B}} T \ll 0.15\right.$ $\mathrm{eV}$ ) to enable hole states that allows the high-frequency transition. As the result, there only leaves the $1 \mathrm{P}_{\mathrm{e}} \rightarrow 1 \mathrm{~S}_{3 / 2}$ transition that may induce the emission at $2.15 \mathrm{eV}$.

In this context, we must take the plasmonic doping of hot electrons into consideration, because (i) the excitation of one plasmon can produce multiple hot electrons[20] (Supplementary Fig. S5); and (ii) the hot electron production has been observed in our plasmonic resonator (Supplementary Fig. S4). These charges, if being injected into the adjacent QDs, can yield a carrier density that 

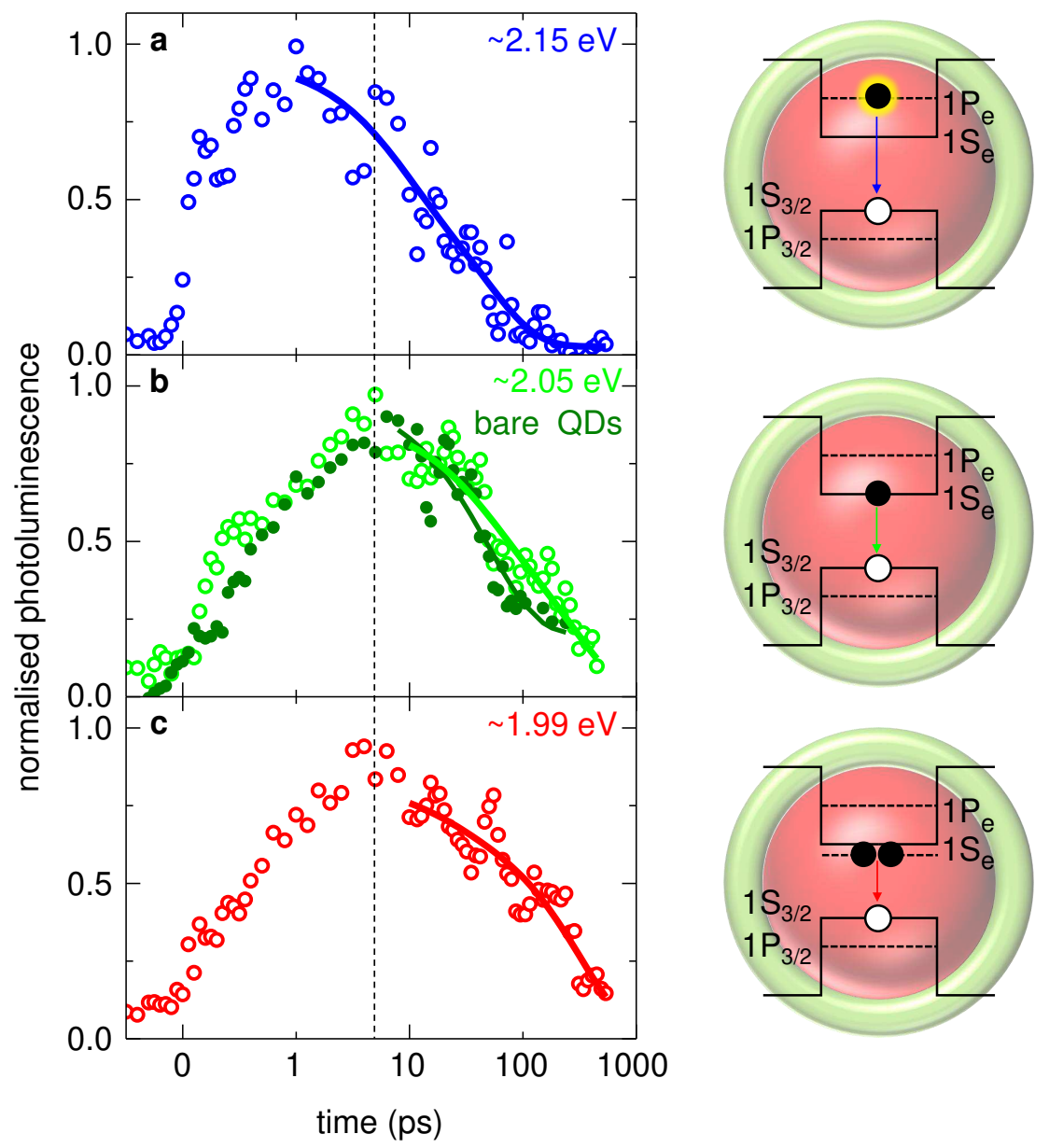

FIG. 4. Relaxations in QDs. Normalised transient PL (open circles) as a function of time from the QDresonator hybrid at $\mathbf{a}, \sim 2.15 \mathrm{eV}, \mathbf{b}, \sim 2.05 \mathrm{eV}$ and $\mathbf{c}, \sim 1.99 \mathrm{eV}$, corresponding to the maxima of the three fitting components in Fig. 3b; the transient PL decay from the bare QD sample (dark green solid circles) is plotted in panel $\mathbf{b}$ as a comparison; the specific energy levels in the QD that the corresponding transitions take place are plotted on the right. The solid curves are the single- (panel $\mathbf{b}$ and $\mathbf{c}$ ) or bi- (panel a) exponential fitted results with parameters demonstrated in Supplementary Table S1, while the dotted vertical line indicates the time point, where the bandedge emission achieves its maximum. The pump fluence is $25.61 \mu \mathrm{J} \mathrm{cm}^{-2}$.

is higher than the absorbed photon density $\left(N_{\mathrm{e}}>N_{\mathrm{ph}}\right)$, thus enabling a high-density population even with a low pump fluence, as illustrated in Fig. 1b. This is not achievable by the Purcell enhancement, in which $N_{\mathrm{e}}=N_{\text {ph }}$ must hold (Fig. 1a). We have calculated the doped carrier density in QDs contacting the surface of Ag lines (Fig. 2b) using[23]:

$$
N_{\mathrm{e}}=\frac{F_{\mathrm{pump}}}{2 c \epsilon_{0}} \cdot\left|\mathbf{E} / \mathbf{E}_{0}\right|^{2} \cdot \frac{1}{\pi^{2}} \frac{e^{2} E_{\mathrm{F}}^{2}}{\hbar} \frac{\hbar \omega-\Delta \phi_{\mathrm{TB}}}{(\hbar \omega)^{4}} \cdot S_{\mathrm{c}}
$$

where $c$ is the light speed, $\epsilon_{0}$ is the vacuum permittivity, $e$ is the electron charge, $E_{\mathrm{F}}$ is the $\mathrm{Ag}$ 
Fermi-level, $\omega$ is the pump frequency, $\left|\mathbf{E} / \mathbf{E}_{0}\right|^{2}$ is the modelled field enhancement, and $F_{\text {pump }}=$ $25.6 \mu \mathrm{J} \mathrm{cm}^{-2}$ is the pump fluence. Here we take $\Delta \phi_{\mathrm{TB}}=1 \mathrm{eV}$ as the tunneling barrier for electrons entering the core-shell QDs (Supplementary Fig. S10), finding that up to 16 - 288 electrons can be doped in each QD when the contact-area $S_{\mathrm{c}}$ between the QD and Ag surface varies from 0.5 to $9 \mathrm{~nm}^{2}$. These are a feasible contact-area range, given that our QDs have a diameter of $7.3 \mathrm{~nm}[25]$. This high-density doping certainly affects carrier relaxations in the QDs. For example, the doped hot electrons can be temporarily accumulated in the conduction band, equivalent to charging the QDs, which can help break the selection rules [29] that forbid the transition between S and P levels[30]. As a result, the $1 \mathrm{P}_{\mathrm{e}} \rightarrow 1 \mathrm{~S}_{3 / 2}$ emission at $\sim 2.15 \mathrm{eV}$ becomes allowed (schematic in Fig. 4a), which is, as discussed above, induced by the plasmonic doping of hot electrons but not the Purcell enhancement.

For further analysis we confine ourselves to QDs' carrier dynamics. In Fig. 4, we have plotted the relaxation of the three main PL components (dashed curves in Fig. 3b fitted using Gaussian functions as in Supplementary Eq.S4). Fig. 4a demonstrates the high-frequency relaxation at $2.15 \mathrm{eV}$, in which the transient PL signal achieves its maximum within $<1$ ps, much earlier than do the transitions at lower frequencies (Fig. $4 \mathbf{b}$ and 4c), and then rapidly decays. This is a clear indication that the doped carriers tend to relax first from the higher but not the lower energy states as the charge injection is a top-down process (Fig. 1b and Supplementary Fig. S6).

Carriers at high-frequency states are unstable, thus quickly relaxing to lower levels, e.g. the bandedge state, which can then induce the $1 S_{e} \rightarrow 1 S_{3 / 2}$ transition (Fig. $4 \mathbf{b}$ and $4 \mathbf{c}$ ) enabling the emission at $\sim 2.05 \mathrm{eV}$ and $\sim 1.99 \mathrm{eV}$ that have similar relaxation tendencies. Here we want to point out that due to the limited time range $(<1000 \mathrm{ps})$ in our PL measurements, we fit the low-frequency (2.05 and $1.99 \mathrm{eV}$ ) decays using single-exponential functions, aiming to recover the fast decay processes; while the slow processes have been captured using Transient Absorption measurements (Supplementary Table. S1). Specifically, we note that the QD-resonator hybrid has a slow-process lifetime of $\tau \sim 3 \mathrm{~ns}$ (Supplementary Fig. S11) in contrast to the $\sim 15 \mathrm{~ns}$ lifetime in the bare QDs[25]. There can be two possibilities for the lifetime reduction: (i) it can be induced by the Purcell enhancement of radative emission, which agrees well with the calculated $F_{\mathrm{P}}$ (Fig. 2b) and the steady-state PL enhancement (Fig. 2d); (ii) it may be due to the non-radiative Auger recombination of trions[28], in which case the QDs must be doped with a number of electrons. Here we want to point out that the PL signals of these two low-frequency components were both measured from a mixture of undoped QDs (where only the Purcell enhancement presents) and doped 
QDs (where both plasmonic doping and the Purcell enhancement take effects), which makes it difficult to explicitly distinguish the contributions from doping or the Purcell enhancement to the shortened lifetime.

To summarize, we have demonstrated emission modification in QDs induced by the charge transfer from an adjacent plasmonic resonator. Specifically, the excitaton of plasmons produces a large number of hot electrons, which transiently dope the QDs, making the excited carrier density larger than the absorbed photon density and hence enabling the spontaneous emission from high-frequency excitonic states. Beyond the scope of the Purcell effect that is limited to LDOS modification, our work has identified a new cavity-emitter interaction pathway for QDs, and by extension for all emitters that can be charged, openning up exciting opportunities in the study of cavity Quantum Electrodynamics and the design of optoelectronic and nanophotonic devices. 


\section{METHODS}

\section{Sample Fabrication}

The grating-like plasmonic resonators have been fabricated by using optical interference lithography[31] together with thermal evaporation. The geometry of the resonator can be seen from Supplementary Fig. S2 and Fig. 2a. The QDs were synthesized using the method detailed in [25], having the CdSe core (diameter of $4 \mathrm{~nm}$ ) covered by a CdS shell (diamter of $7.3 \mathrm{~nm}$ ). The QDs was spin-coated on the resonator, forming a 6-7 layers film on top of the Ag lines.

\section{Steady-state Reflection and PL Measurements}

The angle resolved steady-state reflection and PL have been measured from the samples using the setup presented in Supplementary Fig. S1a. The samples are illuminated by a halogen lamp through an objective (100 times magnification); and the reflected beam is collected through the same objective and then analysed at the Fourier image plane, which allows us to obtain the reflection spectra by scanning optical signals along the Fourier image plane using a fiber that is connected to a spectrometer (OceanOptics USB2000+VIS-NIR-ES). The fiber has a diameter $100 \mu \mathrm{m}$, enabling an angular resolution of $1^{\circ}$. Furthermore, a polarizer was installed before the detection plane to analyse the polarization of the reflected beam. In PL measurements, the halogen lamp was replaced by a laser (532 $\mathrm{nm}$ and power $45 \mathrm{~mW})$.

\section{Transient PL Measurements}

The broadband TPL spectra have been measured using our transient grating PL spectrometer (Supplementary Fig. S1b)[32]. In short, as the result of laser beam (1030 nm, 120 fs duration) interference on a silica film, the refractive index (RI) of the film can be periodically and transiently modulated, forming a grating-like RI distribution that can be used to transiently diffract incoming light. Pumping on the sample with the pulses (515 nm, $120 \mathrm{fs})$, the PL signals are collected and then projected onto the film. Varying the delay time between the PL signals and the transient RI grating, we can get the broadband PL that is transiently diffracted away from the background PL, which are the PL spectra at different time delays. This technique allows us to resolve the broadband PL with a time resolution of $\sim 200$ fs. What's worth mentioning is that the PL signals are collected from a wide angle range $\left(-20^{\circ}\right.$ to $\left.20^{\circ}\right)$ to enhance the PL intensity, thus increasing the signal-to-noise ratio. 


\section{ACKNOWLEDGMENTS}

The authors acknowledge the New Idea Research Funding 2018 (Dodd-Walls Centre for photonic and quantum technologies), the Marsden Fast-start Fund by Royal Society of New Zealand through contract MFP-UOO1827 and MFP-VUW1715 and the Smart Ideas Fund by Ministry of Business, Innovation and Employment, New Zealand through contract UOOX1802. In addition, this work was supported in part by the National Key Research and Development Program of China (No. 2017YFA0205700) and the National Natural Science Foundation of China (Nos. 6192782 and 51861135201), Beijing Municipal Natural Science Foundation (1214027), the Science and Technology Innovation Project of Beijing Institute of Technology and the NSFC funding (12004313). We thank Dr. M. Yan and Dr. F. Hong for their help with thin-film deposition, AFM, and SEM measurements. The authors appreciate Dr. Michael Price for valuable discussions and Dr. Sam Lowrey for help in optical interference lithography and AFM measurements.

\section{Author Contributions}

B.D., K.C. and Y.-H.C. developed the idea; M.I., M. S., Z. H. and P.G. prepared the samples; I.W., M.I., F.L. carried out the optical and other characterization; Y.-H.C. and B.D. performed the simulation; B.D., K.C. Y.-H.C., M.Q., R.J.B., J.M.H. supervised the project; B.D. and Y.-H.C. prepared the manuscript; all authors discussed and analyzed the results.

\section{Competing Interests}

The authors declare no competing interests.

\section{Additional Information}

Supplimentary Information is available for this paper. Correspondence and requests for materials should be addressed to B.D.

\footnotetext{
* These authors contributed equally

$\dagger$ boyang.ding@coherent.com.au

‡ kai.chen@vuw.ac.nz

§qiu_lab@westlake.edu.cn
}

[1] Pelton, M. Modified spontaneous emission in nanophotonic structures. Nat. Photon. 9, 427-435 (2015). .

[2] Purcell, E. M. Spontaneous emission probabilities at radio frequencies. Physical Review 69, 681 
(1946).

[3] Wu, K., Chen, J., Mcbride, J. R. \& Lian, T. Efficient hot-electron transfer by a plasmon-induced interfacial charge-transfer transition. Science 349, 3584-3588 (2015).

[4] Wu, K., Park, Y. S., Lim, J. \& Klimov, V. I. Towards zero-threshold optical gain using charged semiconductor quantum dots. Nature Nanotechnology 12, 1140-1147 (2017). .

[5] Lim, J., Park, Y. S. \& Klimov, V. I. Optical gain in colloidal quantum dots achieved with direct-current electrical pumping. Nature Materials 17, 42-48 (2018).

[6] Kozlov, O. V. et al. Sub-single-exciton lasing using charged quantum dots coupled to a distributed feedback cavity. Science 675, 672-675 (2019).

[7] Tang, J. et al. Colloidal-quantum-dot photovoltaics using atomic-ligand passivation. Nature Materials 10, 765-771 (2011).

[8] Huang, J., Mulfort, K. L., Du, P. \& Chen, L. X. Photodriven charge separation dynamics in $\mathrm{CdSe} / \mathrm{ZnS}$ core/shell quantum dot/cobaloxime hybrid for efficient hydrogen production. Journal of the American Chemical Society 134, 16472-16475 (2012).

[9] Ye, Y. et al. Monolayer excitonic laser. Nature Photonics 9, 733-737 (2015). . 1503.06141.

[10] Jiang, X. F., Zou, C. L., Wang, L., Gong, Q. \& Xiao, Y. F. Whispering-gallery microcavities with unidirectional laser emission. Laser and Photonics Reviews 10, 40-61 (2016).

[11] Lodahl, P., Driel, A. F. V., Nikolaev, I. S. \& Irman, A. Controlling the dynamics of spontaneous emission from quantum dots by photonic crystals. Nature 430, 654 (2004).

[12] Chikkaraddy, R. et al. Single-molecule strong coupling at room temperature in plasmonic nanocavities. Nature 535, 127-130 (2016). .

[13] Chen, X. et al. Mode Modification of Plasmonic Gap Resonances induced by Strong Coupling with Molecular Excitons. Nano Lett. 17, 3246-3251 (2017).

[14] Qin, J. et al. Revealing Strong Plasmon-Exciton Coupling Between Nano-gap Resonators and TwoDimensional Semiconductors at Ambient Conditions. Physical Review Letters 124, 63902 (2020). . 1811.01598 .

[15] Ringler, M. et al. Shaping Emission Spectra of Fluorescent Molecules with Single Plasmonic Nanoresonators. Phys. Rev. Lett. 100, 203002 (2008). .

[16] Ureña, E. B. et al. Excitation enhancement of a quantum dot coupled to a plasmonic antenna. Advanced Materials 24, 314-320 (2012).

[17] Akselrod, G. M. et al. Probing the mechanisms of large Purcell enhancement in plasmonic nanoan- 
tennas. Nat. Photon. 8, 835-840 (2014). .

[18] Ding, B., Hrelescu, C., Arnold, N., Isic, G. \& Klar, T. A. Spectral and directional reshaping of fluorescence in large area self-assembled plasmonic-photonic crystals. Nano Lett. 13, 378-86 (2013).

[19] Ding, B. et al. Tunable Valley Polarized Plasmon-Exciton Polaritons in Two-Dimensional Semiconductors. ACS Nano 13, 1333-1341 (2019). .

[20] Khurgin, J. B. Fundamental limits of hot carrier injection from metal in nanoplasmonics. Nanophotonics 9, 453-471 (2020). 1910.01886.

[21] Clavero, C. Plasmon-induced hot-electron generation at nanoparticle/metal-oxide interfaces for photovoltaic and photocatalytic devices. Nature Photonics 8, 95-103 (2014).

[22] Brongersma, M. L., Halas, N. J. \& Nordlander, P. Plasmon-induced hot carrier science and technology. Nature Nanotechnology 10, 25-34 (2015).

[23] Chen, Y.-h. et al. Bandgap control in two-dimensional semiconductors via coherent doping of plasmonic hot electrons. Nature Communications 12, 4332 (2021). .

[24] Drijvers, E. et al. Revisited Wurtzite CdSe Synthesis: A Gateway for the Versatile Flash Synthesis of Multishell Quantum Dots and Rods. Chemistry of Materials 28, 7311-7323 (2016).

[25] Bisschop, S., Geiregat, P., Aubert, T. \& Hens, Z. The Impact of Core/Shell Sizes on the Optical Gain Characteristics of CdSe/CdS Quantum Dots. ACS Nano 12, 9011-9021 (2018).

[26] Achermann, M., Hollingsworth, J. A. \& Klimov, V. I. Multiexcitons confined within a subexcitonic volume: Spectroscopic and dynamical signatures of neutral and charged biexcitons in ultrasmall semiconductor nanocrystals. Physical Review B - Condensed Matter and Materials Physics 68, 1-5 (2003). 0309712.

[27] Bonati, C. et al. Spectral and dynamical characterization of multiexcitons in colloidal CdSe semiconductor quantum dots. Physical Review B - Condensed Matter and Materials Physics 71, 1-6 (2005).

[28] Pietryga, J. M. et al. Spectroscopic and device aspects of nanocrystal quantum dots. Chemical Reviews 116, 10513-10622 (2016).

[29] Park, S. H. et al. Nonlinear optical properties of quantum-confined CdSe microcrystallites. Journal of the Optical Society of America B 7, 2097 (1990).

[30] Ekimov, A. I. et al. Absorption and intensity-dependent photoluminescence measurements on CdSe quantum dots: assignment of the first electronic transitions: erratum. Journal of the Optical Society of America B 11, 524 (1993). 
[31] Lowrey, S., Bourke, L., Ding, B. \& Blaikie, R. Ultrahigh NA, high aspect ratio interference lithography with resonant dielectric underlayers. J. Vac. Sci. Technol. B 32, 2166-2746 (2014).

[32] Chen, K., Gallaher, J. K., Barker, A. J. \& Hodgkiss, J. M. Transient grating photoluminescence spectroscopy: An ultrafast method of gating broadband spectra. Journal of Physical Chemistry Letters 5, 1732-1737 (2014). 


\section{Supplementary Files}

This is a list of supplementary files associated with this preprint. Click to download.

- S.IDingetal.pdf 